\title{
Higgs production through vector-boson fusion at the NLO matched with parton showers
}

\author{
Stefano Frixione ${ }^{\mathrm{a}, \mathrm{b}}$, Paolo Torrielli ${ }^{\mathrm{c}, *}$, Marco Zaro ${ }^{\mathrm{d}}$ \\ a PH Department, TH Unit, CERN, CH-1211 Geneva 23, Switzerland \\ b ITPP, EPFL, CH-1015 Lausanne, Switzerland \\ c Institut für Theoretische Physik, Universität Zürich, Winterthurerstrasse 190, CH-8057 Zürich, Switzerland \\ d Centre for Cosmology, Particle Physics and Phenomenology (CP3), Université Catholique de Louvain, Chemin du Cyclotron 2, B-1348 Louvain-la-Neuve, Belgium
}

\section{A R T I C L E I N F O}

\section{Article history:}

Received 14 May 2013

Accepted 11 August 2013

Available online $\mathrm{xxxx}$

Editor: G.F. Giudice

\section{Keywords:}

QCD phenomenology

Higgs

LHC

\begin{abstract}
A B S T R A C T
We present a study of Higgs hadroproduction through vector-boson fusion at the NLO in QCD matched with parton showers. We discuss the matching systematics affecting this process through a comparison of the aMC@NLO predictions with the POWHEG and the pure-NLO ones.
\end{abstract}

(C) 2013 Elsevier B.V. All rights reserved.

\section{Introduction}

The production of a Standard-Model (SM) Higgs boson $\left(H^{0}\right)$ through the so-called vector-boson-fusion (VBF) mechanism features the second-largest cross section among the $H^{0}$ production channels in hadronic collisions and, although smaller than the gluon-fusion one by about one order of magnitude, it still provides useful complementary informations. After the discovery of a SMHiggs-like particle [1,2], the emphasis is rapidly shifting towards the determination of its properties, and in this respect VBF may play an increasingly important role, owing to its sensitivity to various combinations of Higgs couplings [3], which can be studied by considering different decay channels. However, the very distinctive features of VBF, with two jets lying relatively close to the beam line and travelling in opposite directions, render it a challenging case, given that rather severe cuts have to be applied in order to reduce backgrounds (among which, in the coupling measurement perspective, one may count the contamination due to $g g \rightarrow H^{0}$ ). While the typical kinematic regions probed at the LHC do not pose problems for perturbative-QCD computations (as shown by the behaviour of the rather moderate NLO [4-6] and NNLO [7,8] corrections in parton-level results), the presence of two jets in a hadronically-enriched environment implies the necessity of using hadron-level simulations such as those generated with parton

\footnotetext{
* Corresponding author.

E-mail address: torriell@physik.uzh.ch (P. Torrielli).
}

shower Monte Carlo's (PSMC's), in order to obtain more realistic predictions.

It has by now become a rather standard procedure that of matching NLO QCD results with PSMC's, by using either the MC@NLO [9] or the POWHEG [10,11] formalism. Because of the potential importance of shower and hadronisation effects and of the good behaviour of NLO corrections, VBF appears in fact to be an ideal application for matching techniques. However, this has been done so far only in the context of the POWHEG approach [12]; in this Letter, we amend this by presenting MC@NLO results obtained with the fully-automated aMC@NLO framework, and by comparing them extensively with those obtained with the code constructed in reference [12] and implemented in the publicly available POWHEG-Box framework [13]. The primary motivation for doing so is phenomenological. As is known, MC@NLO and POWHEG differ by terms of order $\mathcal{O}\left(\alpha_{S}^{b+2}\right)$ [14], i.e. two orders larger than the Born's; furthermore, they differ by logarithmic orders beyond the leading even if matched to the same PSMC, owing to the fact that POWHEG generates the first emission with own Sudakov form factors, independent of those of the PSMC. ${ }^{1}$ While these differences are typically small, consistently with their being beyond the nominal accuracy of the calculations, Higgs production in gluon fusion constitutes a striking counter-example, with the two approaches yielding significant discrepancies in the Higgs

\footnotetext{
1 The latter differences are actually logarithmically leading in the case of an angular-ordered PSMC which does not include a vetoed-truncated shower [10].
} 


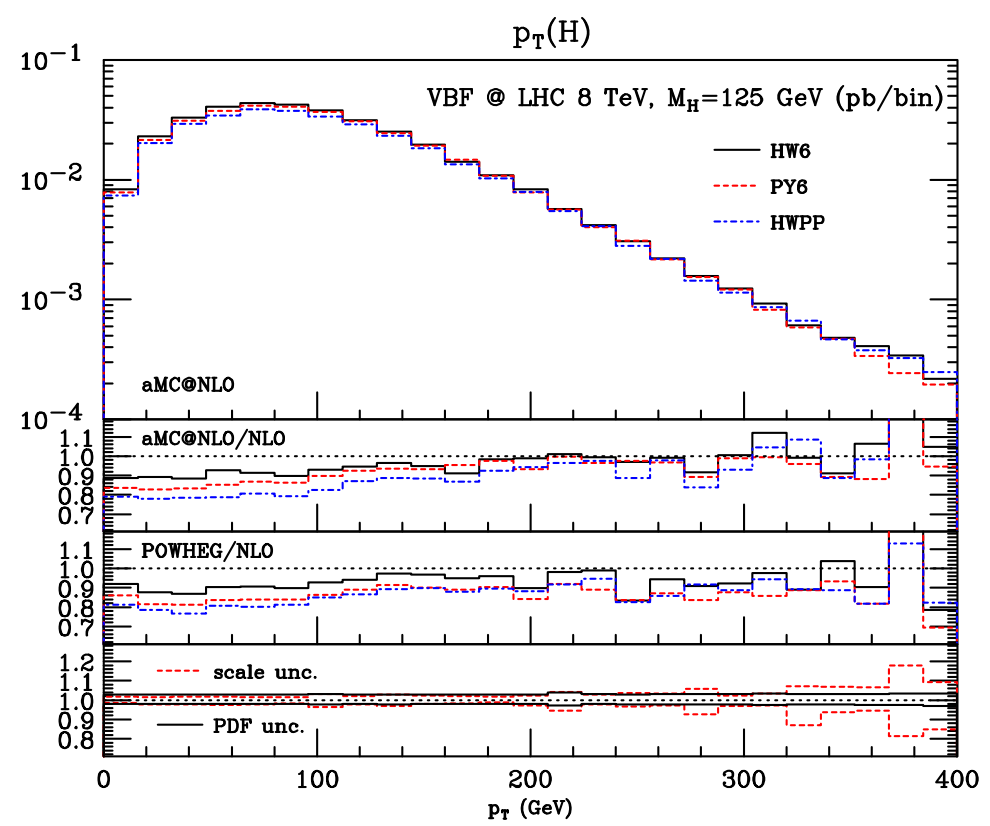

$\mathrm{y}(\mathrm{H})$

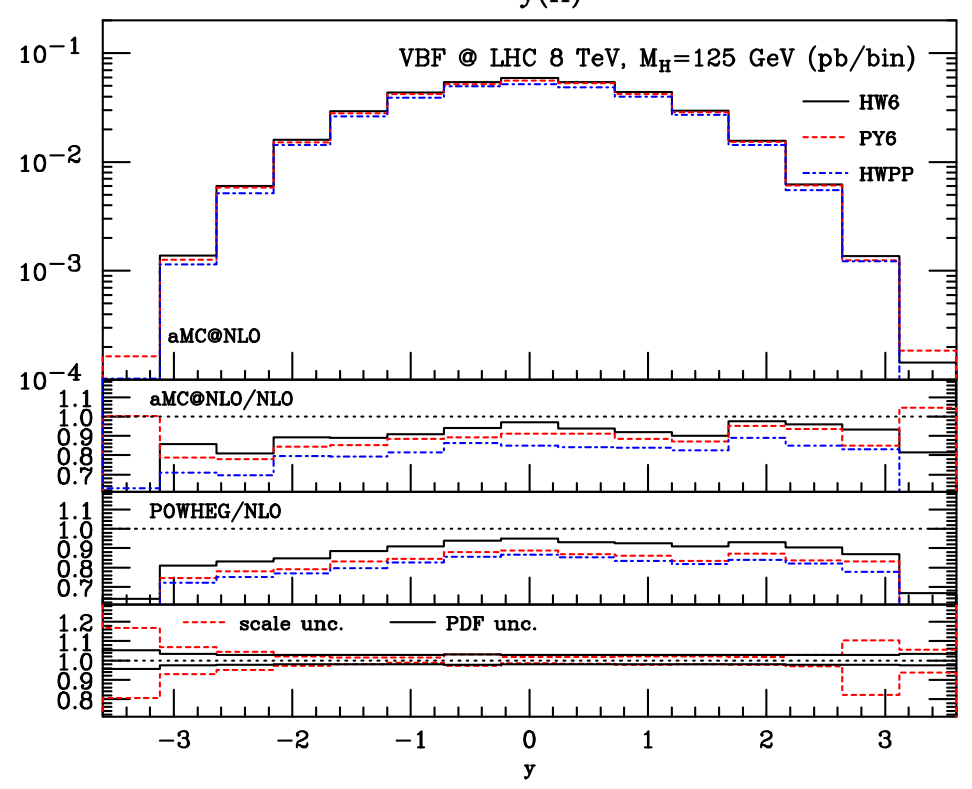

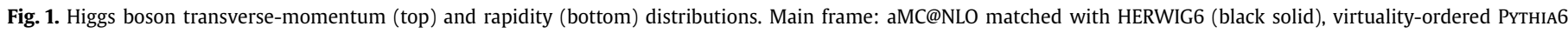

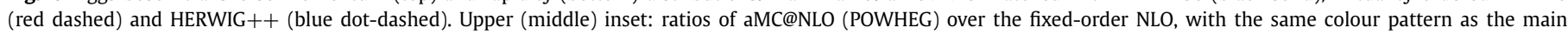
frame. Lower inset: scale (red-dashed) and PDF (black solid) uncertainties for aMC@NLO + HERWIG6. See text for further details.

transverse momentum, ${ }^{2}$ and in the Higgs-hardest-jet rapidity difference. The latter observable in particular, being quite sensitive to the radiation pattern generated by the $\mathrm{PSMC}^{3}$ plus the internal Sudakov in the case of POWHEG, could have direct implications for VBF, given the importance of 'extra' radiation in this process. In general, the differences between the MC@NLO and POWHEG results should give one a fair idea of the NLO-matching systematics, a topic which, to the best of our knowledge, has not been studied in VBF Higgs production. A lesser motivation is technical, and is that of validating the aMC@NLO machinery with a further

\footnotetext{
2 Before any tuning of the hfact parameter in POWHEG.

3 See Ref. [15] for a discussion on this point.
}

non-trivial process on top of those considered so far. We remind the reader that aMC@NLO is a generator that implements the matching of a generic NLO QCD computation with a PSMC according to the MC@NLO formalism; its defining feature is that all ingredients of such matching and computation are fully automated. The program is developed within the MADGRAPH 5 [16] framework and, as such, it does not necessitate of any coding by the user, the specification of the process and of its basic physics features (e.g. particle masses or phase-space cuts) being the only external informations required: the relevant computer codes are then generated on-the-fly, and the only practical limitation is represented by CPU availability. aMC@NLO is based on different building blocks, each devoted to the generation and evaluation of a specific contribution to an NLO-matched computation. MADFKS [17] 

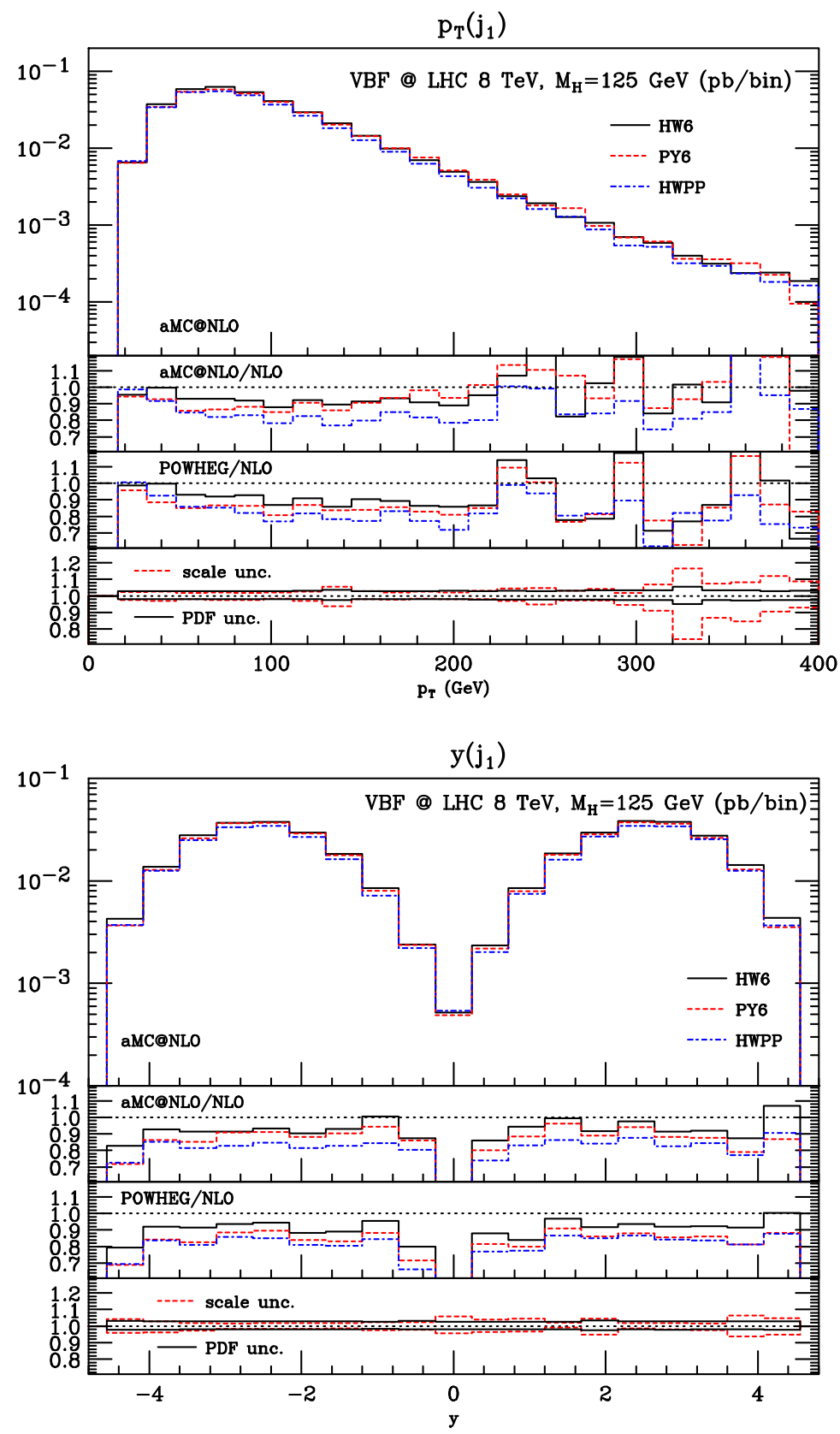

Fig. 2. Same pattern as in Fig. 1; for the hardest-jet transverse momentum (top) and rapidity (bottom).

deals with the Born and real-emission terms, and in particular it performs, according to the FKS prescription [18,19], the subtraction of the infrared singularities that appear in the latter matrix elements; moreover, it is also responsible for the processindependent generation of the so-called Monte Carlo subtraction terms, namely the contributions that prevent any double-counting in the MC@NLO cross sections. Finally, MAdLoop [20] computes the finite part of the virtual contributions, using the OPP [21] one-loop integrand-reduction method and its implementation in CuTTOoLs [22].

\section{Results}

In this section we present results relevant to the production of a $125 \mathrm{GeV}$ Standard-Model Higgs boson through a VBF mechanism at the $8 \mathrm{TeV}$ LHC. aMC@NLO includes all interferences between $t$ - and $u$-channel diagrams, such as those occurring for same-flavour quark scattering and for partonic channels that can be obtained by the exchange of either a $Z^{0}$ or a $W^{ \pm}$boson (e.g. $u d \rightarrow H^{0} u d$ ). These interferences, which are kinematically suppressed and de facto negligible, are not included in POWHEG. Furthermore, only vertex loop-corrections are considered in both matching schemes, as the omitted loops are totally negligible [23]. Electroweak NLO corrections, negative and of the order of $5 \%$ for this Higgs mass and collider energy $[23,24]$, are not included. The Higgs boson is considered as stable.

Matching with different showers, namely HERWIG6 [25], HERWIG++ [26], and virtuality-ordered PYTHIA6 [27] (abbreviated in the following with HW6, HWPP, and PY6, respectively), is considered both in aMC@NLO and in POWHEG, in order to estimate the dependence of physics results on the shower model, within the same matching scheme. 


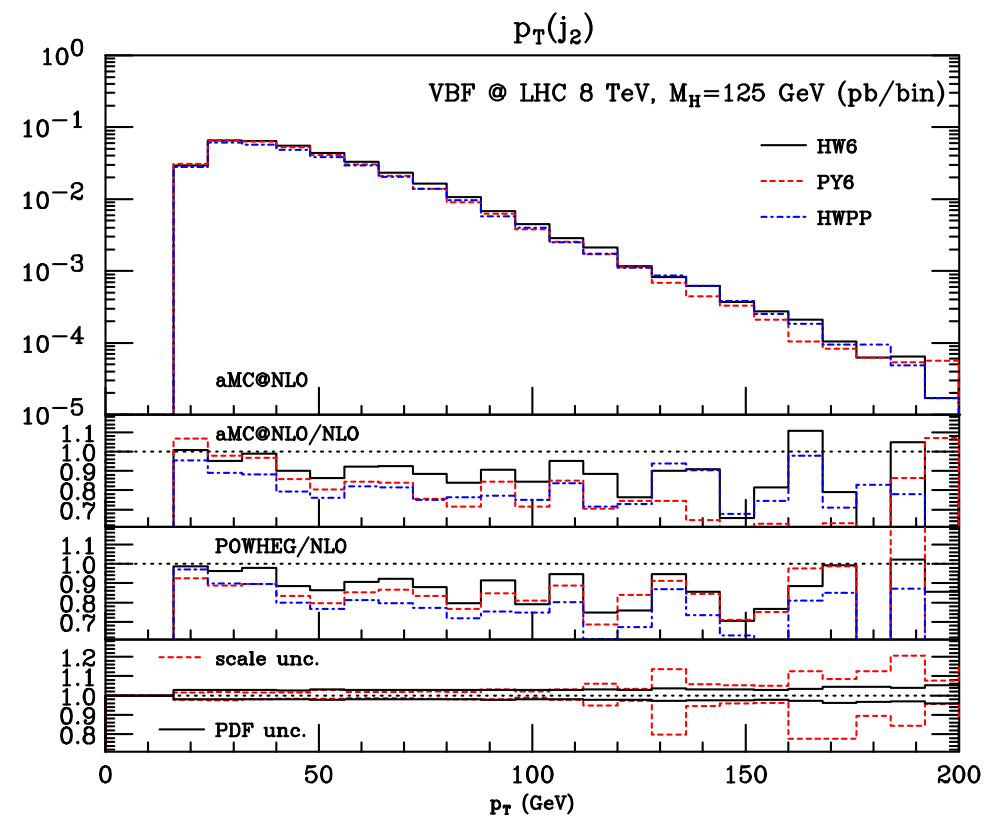

$\mathrm{y}\left(\mathrm{j}_{2}\right)$

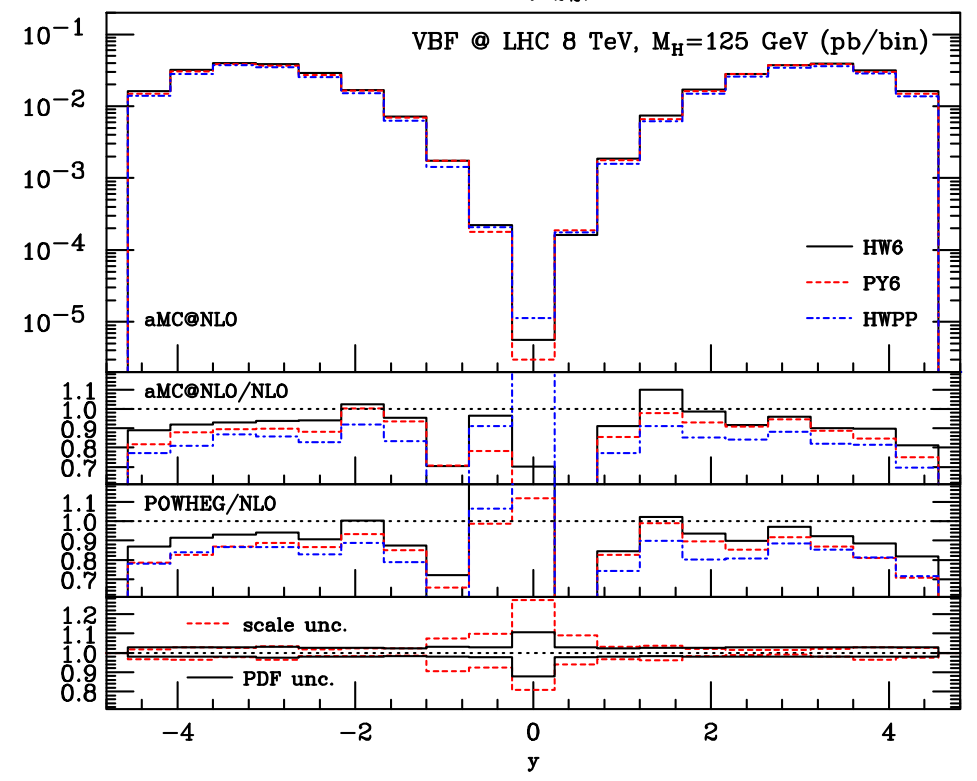

Fig. 3. Same pattern as in Fig. 1; for the second hardest-jet transverse momentum (top) and rapidity (bottom).

\subsection{Setup: parameters and cuts}

Here we list the input settings employed in this computation. The values for the Standard-Model parameters follow the prescriptions of the Higgs Cross-Section Working Group (HXSWG) [28]:

$M_{W}=80.398 \mathrm{GeV}, \quad \Gamma_{W}=2.089 \mathrm{GeV}$,

$M_{Z}=91.188 \mathrm{GeV}, \quad \Gamma_{Z}=2.496 \mathrm{GeV}$,

$G_{F}=1.166 \times 10^{-5} \mathrm{GeV}^{-2}$.

Results are obtained by using the MSTW2008NLO PDF set [29], with errors estimated at the $68 \%$ confidence level. Moreover, renormalisation and factorisation scales are set equal to the $W$ mass, as suggested by the HXSWG. All parton showers are run with their default settings, with the only exception of aMC@NLO + PY6, where PARP (67) and PARP (71) are set equal to one. Furthermore, no simulation of the underlying event is performed.

Parton-level events are generated without imposing generation cuts, with the exception of a technical cut that requires at least two jets with $p_{T}(j)>2 \mathrm{GeV}$ in the aMC@NLO samples. This cut has been extensively checked not to introduce any bias in total rates and differential distributions. After shower and hadronisation, typical selection cuts used in experimental VBF analyses (called VBF cuts henceforth) are applied: hadrons are clustered into jets by using the anti- $k_{T}$ algorithm [30] as implemented in FASTJET [31], with $\Delta R=0.5$. The presence of at least two jets is required, with $p_{T}(j)>20 \mathrm{GeV}$ and $|y(j)|<4.5$. Furthermore, the two hardest jets (i.e. the two jets with the largest transverse momenta) among those fulfilling these criteria are required to have an invariant mass $M\left(j_{1}, j_{2}\right)>600 \mathrm{GeV}$ and a rapidity separation $\left|\Delta y\left(j_{1}, j_{2}\right)\right|>4$. 

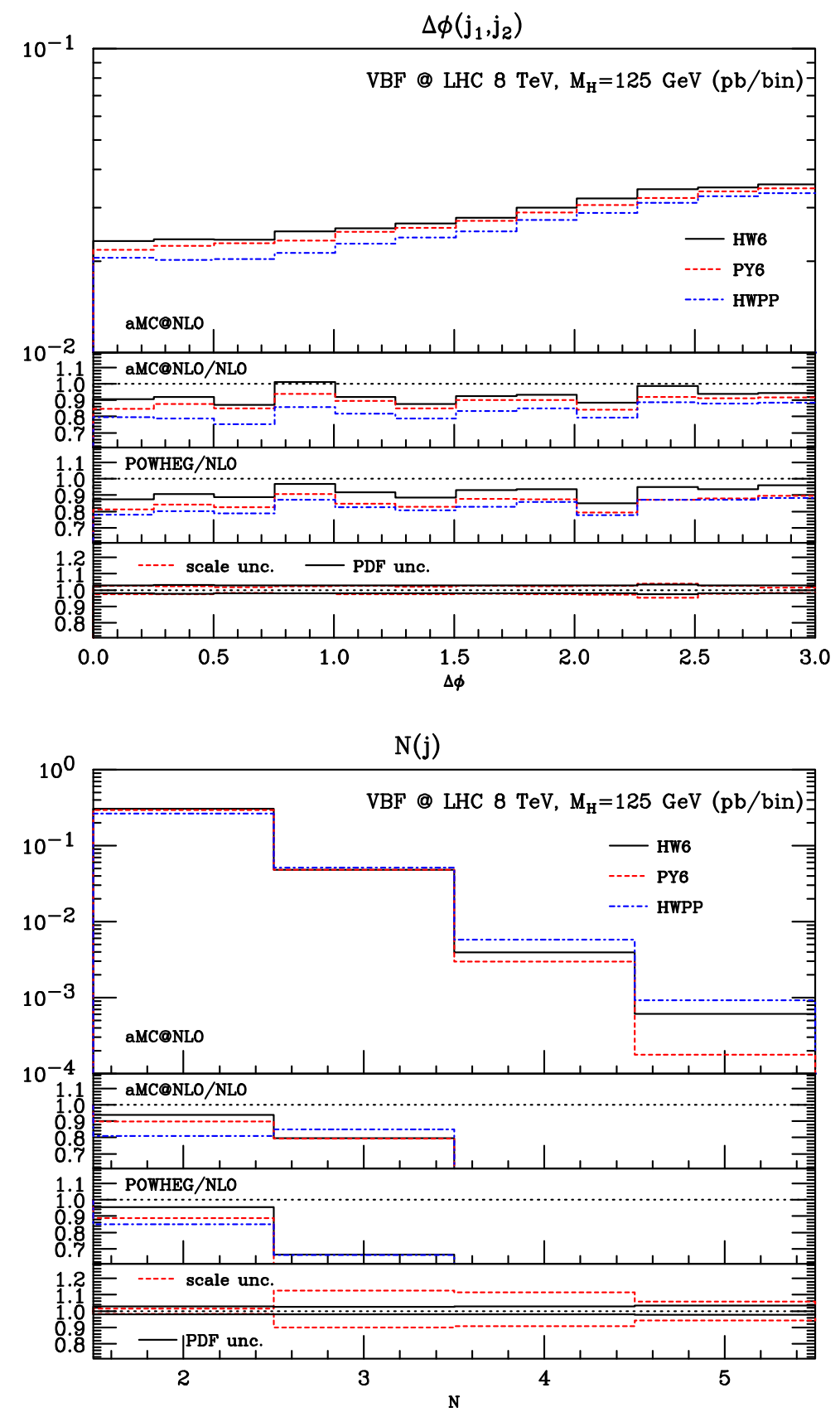

Fig. 4. Same pattern as in Fig. 1; for the azimuthal separation of the two hardest jets (top) and the exclusive jet-multiplicities (bottom).

\subsection{Differential distributions}

We now present results for various differential distributions. The same pattern is adopted for all figures. In the main frame of each figure, the three curves that correspond to the aMC@NLO samples are shown: black solid for HW6, red dashed for PY6, and blue dot-dashed for HWPP. The upper and central insets show, with the same colours and patterns as the main frame, the ratios of the aMC@NLO and POWHEG results over the fixed-order NLO ones, ${ }^{4}$ in order to assess the impact of the different parton showers and matching schemes on the observables considered. The lower insets show the scale (red dashed) and PDF (black solid)

4 Also computed with aMC@NLO and cross-checked against existing results. uncertainties relevant to the aMC@NLO + HW6 sample. The scalevariation band is the envelope of the results obtained by varying independently the factorisation and renormalisation scales in the ranges

$\frac{M_{W}}{2}<\mu_{R}, \quad \mu_{F}<2 M_{W}$,

while the PDF errors are computed with the Hessian method [32], as prescribed by the MSTW set. We remind the reader that the aMC@NLO Les Houches parton-level event files store additional information sufficient to the automatic determination of scale and PDF uncertainties at no extra CPU cost, by means of the reweighting technique presented in [33].

Part of the differences that will appear in the upper and middle insets is due to the different impact of the VBF cuts on the 

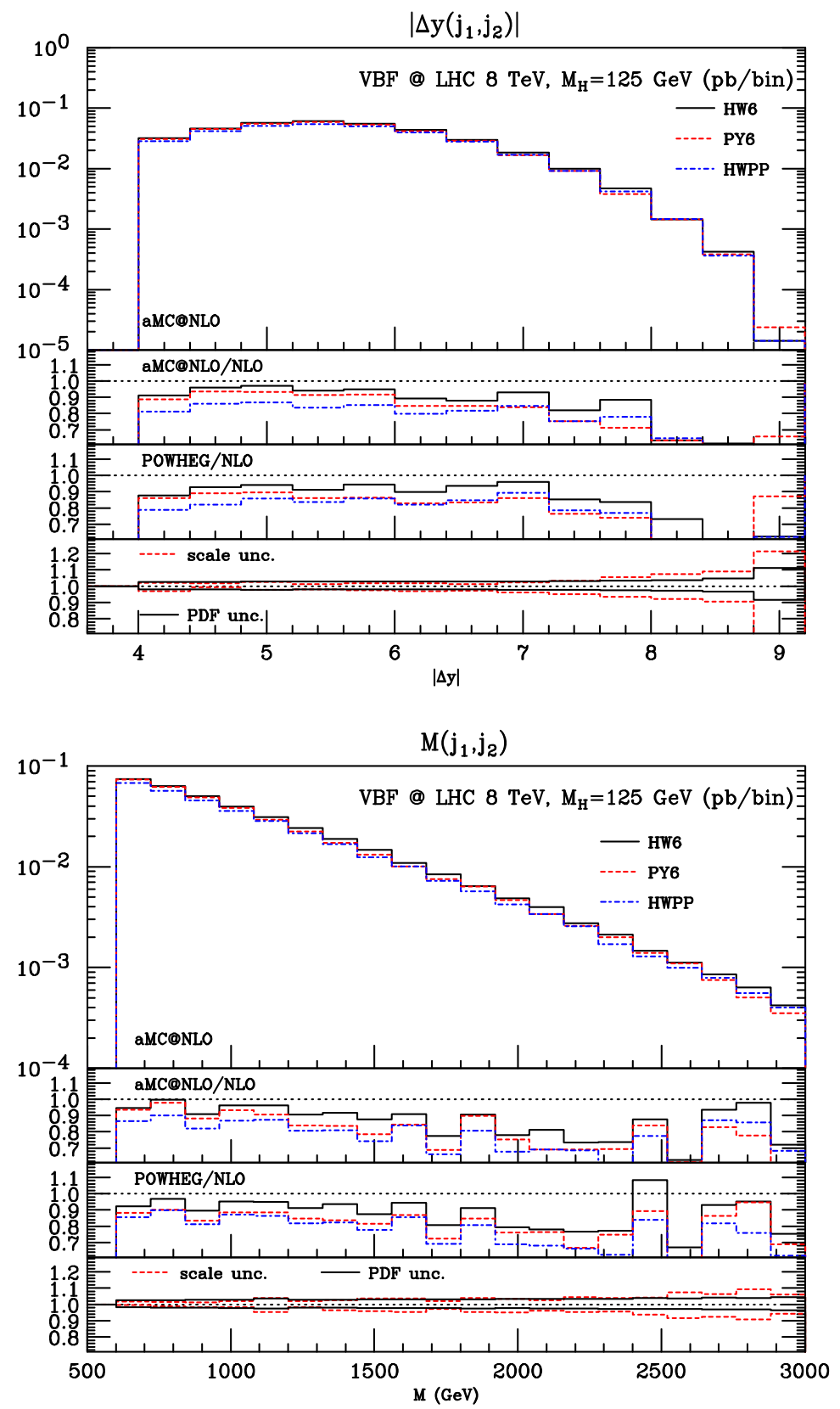

Fig. 5. Same pattern as in Fig. 1; for the rapidity separation (top) and invariant mass (bottom) of the two hardest jets.

Table 1

Ratios of matched-NLO cross sections to the fixed-order NLO one after VBF cuts for aMC@NLO and POWHEG.

\begin{tabular}{llll}
\hline & HERWIG6 & PYTHIA6 & HERWIG ++ \\
\hline aMC@NLO & 0.93 & 0.89 & 0.83 \\
POWHEG & 0.92 & 0.86 & 0.83 \\
\hline
\end{tabular}

QCD radiation generated by the various PSMC's. To better understand this effect, in Table 1 we quote the ratios of the matchedNLO cross section after VBF cuts to the fixed-order NLO one, $\sigma_{\text {CUTS }}^{\text {NLO }}=0.388(2) \mathrm{pb}$. It can be highlighted that these ratios are all smaller than one, as typically parton showers tend to spread the radiation hardness throughout the phase space, causing slightly more events to fail the cuts. On top of this, there is a clear pattern $\sigma_{\text {CUTS }}^{\text {HW6 }}>\sigma_{\text {CUTS }}^{\text {PY6 }}>\sigma_{\text {CUTS }}^{\text {HWPP }}$ both for aMC@NLO and POWHEG.
In Figs. 1-3 we show the transverse momentum and rapidity of the Higgs boson and of the two hardest (tagging) jets. All these observables are described with NLO accuracy, as they are non-trivial in their full kinematic ranges already at the Born level $\mathcal{O}\left(\alpha_{S}^{0}\right)$. Therefore, general agreement among the two different matching frameworks, as well as among different showers is expected. Indeed, all NLO-matched curves are fairly compatible with each other once the ratios in Table 1 and the theoretical uncertainties in the lower insets are taken into account. The comparison with the fixed-order NLO prediction, on top of the overall normalisation effect already shown in Table 1, displays a consistent action of the shower in affecting the jet spectra, an effect which is increasingly important as one moves downwards in the jet hierarchy (i.e. from the hardest to the softest jets); in fact this trend will become even more evident in the case of the third jet (see later). As a consequence of the recoil against the shower-enriched jet activity, 

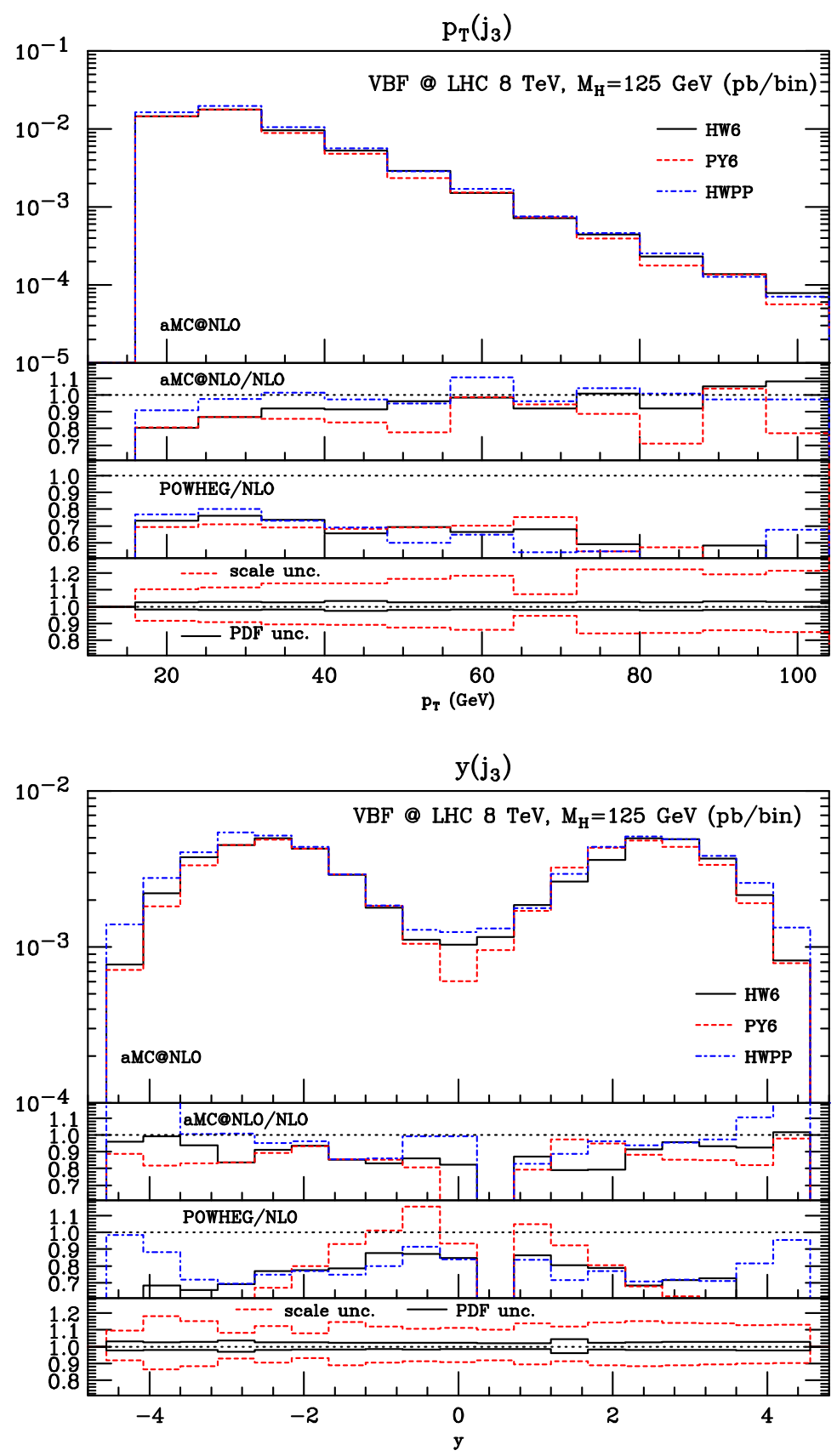

Fig. 6. Same pattern as in Fig. 1; for the third hardest-jet transverse momentum (bottom) and rapidity (top).

NLO-matched curves display harder and more central Higgs-boson distributions. For the observables shown in these figures, PDF and scale uncertainties are generally small (typically of the order of $\pm 3 \%$ to $\pm 5 \%$ ), and fairly constant, with only mild increases at large transverse momenta.

Similar conclusions as the ones presented above can be drawn for the azimuthal separation between the two tagging jets, displayed in the top plot of Fig. 4, which also shows excellent shape agreement between fixed-order and matched computations. This is reassuring, since this observable is particularly sensitive to Higgsboson quantum numbers as spin and parity [34-36], and therefore any theoretical uncertainty is reflected on the characterisation of Higgs properties.

Showering effects are more important for observables such as the invariant mass and the rapidity separation of the two hardest jets. These quantities, shown in Fig. 5, can probe extreme kinematic configurations, where the two jets lie at very large rapidities in opposite hemispheres. At fixed-order and at the parton level, events with large invariant mass and rapidity separation involve partons with energies of up to $\mathcal{O}(1 \mathrm{TeV})$. Such energetic partons, when processed through the shower, have a large probability to fragment several times, so that the resulting hadron-level jets may carry only a small fraction of their energy. This results in the $20 \%$ - to $30 \%$-deficit, with respect to the fixed-order NLO, visible in the matched curves at the rightmost edge of these distributions. In the same region, the theoretical uncertainties grow up to the level of $\pm 10-15 \%$, especially at large rapidity difference. For the PDF's this corresponds to the larger uncertainty at $x \sim 1$, whereas for the scale uncertainty this can be understood as the inadequacy of the choice $\mu_{R, F}=M_{W}$ for such extreme kinematics. 

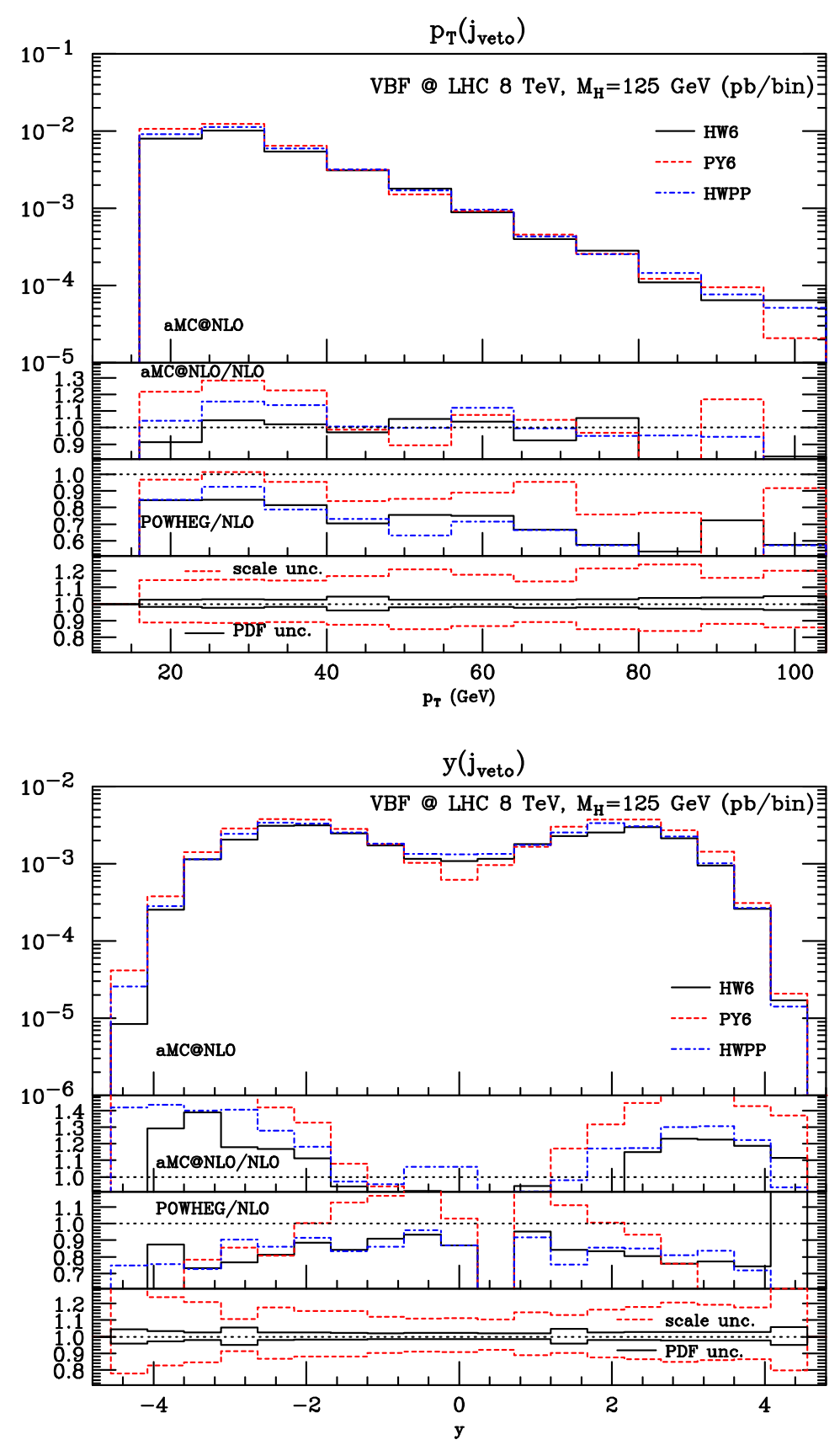

Fig. 7. Same pattern as in Fig. 1; for the veto-jet transverse momentum (top) and rapidity (bottom).

Indeed we have checked that, by employing a dynamical scale like $\mu_{R, F}=\sum_{i} p_{T, i} / 2$ (the sum running over final-state partons), the increase in the scale-uncertainty band at large rapidity difference is very much reduced, to the level of $\pm 5 \%$, with a negligible shift in the central value.

Observables relevant to the third-hardest jet are more sensitive to the different matching procedures and to the effects of parton showers, since their description at the matrix-element level is only LO. In the lower plot of Fig. 4 the exclusive jet-multiplicity is shown. While the 2-jet bin closely follows the ratios in Table 1, the 3 -jet bin shows larger differences, with POWHEG predicting less events than aMC@NLO. The deficit with respect to the fixed-order result in this bin ranges from 15\% to 20\% for aMC@NLO, whereas it is larger, $30 \%$ or more, for POWHEG, irrespectively of the PSMC employed. This indicates that such an effect is mainly a matching systematics rather than being induced by different shower models. It has to be stressed, however, that the POWHEG and aMC@NLO predictions are still compatible within scale uncertainties, which for the 3 -jet bin are about $\pm 10 \%$, consistently with the LO precision of this observable. From the 4 -jet bin onwards, the description is completely driven by the leading-logarithmic (LL) accuracy of the showers and by the tunes employed, which translates in large differences among the various generators. For such jet multiplicities, theoretical-uncertainty bands are completely unrepresentative.

The 3-jet-bin pattern described above determines the normalisation of the third-jet transverse-momentum and rapidity distributions, shown in Fig. 6. These variables can be significantly affected by the different radiation produced by the PSMC's. In particular the aMC@NLO results, which are quite close to the pure NLO on average, display a $\pm 15 \%$ dependence on the shower adopted. 
Conversely, the three POWHEG predictions are slightly closer to each other; we reckon that this is a consequence of the hardest emission being generated in this formalism by a Sudakov which is independent of the actual PSMC employed, as already mentioned in the introduction. The POWHEG curves show a 30\%-deficit with respect to the pure NLO, and are more central and softer than the aMC@NLO ones. Different settings in PYTHIA6 have been checked to induce a variation in the results within the previously mentioned discrepancy range, which is thus to be considered as a genuine measure of the matching systematics affecting these quantities. Scale uncertainties are again compatible with the LO nature of these observables, of the order of $\pm 10 \%$ in the whole rapidity range, and growing from $\pm 10 \%$ to $\pm 20 \%$ with transverse momentum.

Because of the peculiar radiation pattern of VBF, which favours QCD emissions far from the central-rapidity region, one way to reduce the contamination due to background processes, as well as from other production channels (e.g. gluon fusion), is that of rejecting any event featuring a veto jet, namely an extra jet with rapidity lying between those of the two hardest jets:

$\min \left\{y\left(j_{1}\right), y\left(j_{2}\right)\right\}<y\left(j_{\text {veto }}\right)<\max \left\{y\left(j_{1}\right), y\left(j_{2}\right)\right\}$.

The predictions for the transverse momentum and rapidity of the veto jet are shown in Fig. 7. The definition in Eq. (3) implies that the more central the third jet, the larger the probability that it be the veto jet. Since POWHEG predicts a more central third jet with respect to aMC@NLO, the veto condition has the effect that the two predictions for the veto jet are slightly closer to each other than for the third jet. aMC@NLO yields visibly softer and less central distributions, with discrepancies of $20 \%$ to $30 \%$ with respect to the pure NLO at small transverse momentum and large rapidity. The POWHEG predictions for the transverse momentum are softer than aMC@NLO (with the exception of the matching to PY6, where shapes are similar), while rapidities are more central, with $20 \%$ to $30 \%$-discrepancies with respect to the pure NLO at the edges of the spectra. As was the case for the third-hardest jet, the observables related to the veto jet are described only at LO accuracy, and affected by large uncertainties, roughly $\pm 15 \%$.

\section{Conclusions}

We have presented a comparison between aMC@NLO, POWHEG, and fixed-order-NLO predictions for VBF Higgs production at the $8 \mathrm{TeV}$ LHC. This study allows one to asses the NLO-matching systematics affecting this process and its various key-observables. Our results can be summarised as follows. For observables described with NLO accuracy at the parton level, predictions display small theoretical uncertainties, up to $\pm 5 \%$, and show a good agreement between the two matching schemes. Differences with respect to the pure-NLO predictions result from the action of the shower on the jet activity of the events. For quantities described with LO precision, theoretical uncertainties are consistently larger, of the order of $\pm 10 \%$ to $\pm 15 \%$, as well as the discrepancy between the two matching prescriptions, with aMC@NLO generally closer to the fixed-order NLO than POWHEG; in particular there is a visible effect in the observables related to the third jet. Still, all results are largely compatible once theoretical error bands are taken into account.

\section{Acknowledgements}

We are grateful to Fabio Maltoni for many useful comments on the manuscript, and to Carlo Oleari for discussions. S.F. is on leave of absence from INFN, sezione di Genova. This work has been supported in part by the ERC grant 291377 "LHCtheory: Theoretical predictions and analyses of LHC physics: advancing the precision frontier", by the Forschungskredit der Universität Zürich, by the Swiss National Science Foundation (SNF) under contract 200020-138206 and by the Research Executive Agency (REA) of the European Union under the Grant Agreement number PITN-GA2010-264564 (LHCPhenoNet). The work of M.Z. is supported by the IISN "MadGraph" convention 4.4511.10, the IISN "Fundamental interactions" convention 4.4517.08 and the Belgian IAP Program BELSPO P7/37.

\section{References}

[1] G. Aad, et al., Observation of a new particle in the search for the Standard Model Higgs boson with the ATLAS detector at the LHC, Phys. Lett. B 716 (2012) 1-29, http://dx.doi.org/10.1016/j.physletb.2012.08.020, arXiv:1207.7214.

[2] S. Chatrchyan, et al., Observation of a new boson at a mass of $125 \mathrm{GeV}$ with the CMS experiment at the LHC, Phys. Lett. B 716 (2012) 30-61, http://dx.doi.org/ 10.1016/j.physletb.2012.08.021, arXiv:1207.7235.

[3] T. Plehn, D.L. Rainwater, D. Zeppenfeld, Determining the structure of Higgs couplings at the LHC, Phys. Rev. Lett. 88 (2002) 051801, http://dx.doi.org/ 10.1103/PhysRevLett.88.051801, arXiv:hep-ph/0105325.

[4] T. Han, G. Valencia, S. Willenbrock, Structure function approach to vector boson scattering in p p collisions, Phys. Rev. Lett. 69 (1992) 3274-3277, http://dx.doi.org/10.1103/PhysRevLett.69.3274, arXiv:hep-ph/9206246.

[5] T. Figy, C. Oleari, D. Zeppenfeld, Next-to-leading order jet distributions for Higgs boson production via weak-boson fusion, Phys. Rev. D 68 (2003) 073005, http://dx.doi.org/10.1103/PhysRevD.68.073005, arXiv:hep-ph/0306109.

[6] K. Arnold, M. Bahr, G. Bozzi, F. Campanario, C. Englert, et al., VBFNLO: A parton level Monte Carlo for processes with electroweak bosons, Comput. Phys. Commun. 180 (2009) 1661-1670, http://dx.doi.org/10.1016/j.cpc.2009.03.006, arXiv:0811.4559.

[7] P. Bolzoni, F. Maltoni, S. Moch, M. Zaro, Higgs production via vector-boson fusion at NNLO in QCD, Phys. Rev. Lett. 105 (2010) 011801, http://dx.doi.org/ 10.1103/PhysRevLett.105.011801, arXiv:1003.4451.

[8] P. Bolzoni, F. Maltoni, S.-O. Moch, M. Zaro, Vector boson fusion at NNLO in QCD: SM Higgs and beyond, Phys. Rev. D 85 (2012) 035002, http://dx.doi.org/ 10.1103/PhysRevD.85.035002, arXiv:1109.3717.

[9] S. Frixione, B.R. Webber, Matching NLO QCD computations and parton shower simulations, JHEP 0206 (2002) 029, arXiv:hep-ph/0204244.

[10] P. Nason, A new method for combining NLO QCD with shower Monte Carlo algorithms, JHEP 0411 (2004) 040, http://dx.doi.org/10.1088/1126-6708/ 2004/11/040, arXiv:hep-ph/0409146.

[11] S. Frixione, P. Nason, C. Oleari, Matching NLO QCD computations with parton shower simulations: the POWHEG method, JHEP 0711 (2007) 070, http://dx.doi.org/10.1088/1126-6708/2007/11/070, arXiv:0709.2092.

[12] P. Nason, C. Oleari, NLO Higgs boson production via vector-boson fusion matched with shower in POWHEG, JHEP 1002 (2010) 037, http://dx.doi.org/ 10.1007/JHEP02(2010)037, arXiv:0911.5299.

[13] S. Alioli, P. Nason, C. Oleari, E. Re, A general framework for implementing NLO calculations in shower Monte Carlo programs: the POWHEG BOX, JHEP 1006 (2010) 043, http://dx.doi.org/10.1007/JHEP06(2010)043, arXiv:1002.2581.

[14] P. Nason, B. Webber, Next-to-leading-order event generators, Annu. Rev. Nucl. Part. Sci. 62 (2012) 187-213, http://dx.doi.org/10.1146/ annurev-nucl-102711-094928, arXiv:1202.1251.

[15] R. Frederix, S. Frixione, Merging meets matching in MC@NLO, JHEP 1212 (2012) 061, http://dx.doi.org/10.1007/JHEP12(2012)061, arXiv:1209.6215.

[16] J. Alwall, M. Herquet, F. Maltoni, O. Mattelaer, T. Stelzer, MadGraph 5: Going beyond, JHEP 1106 (2011) 128, http://dx.doi.org/10.1007/JHEP06(2011)128, arXiv:1106.0522.

[17] R. Frederix, S. Frixione, F. Maltoni, T. Stelzer, Automation of next-to-leading order computations in QCD: the FKS subtraction, JHEP 10 (2009) 003, http://dx.doi.org/10.1088/1126-6708/2009/10/003, arXiv:0908.4272.

[18] S. Frixione, Z. Kunszt, A. Signer, Three jet cross-sections to next-toleading order, Nucl. Phys. B 467 (1996) 399-442, http://dx.doi.org/10.1016/ 0550-3213(96)00110-1, arXiv:hep-ph/9512328.

[19] S. Frixione, A General approach to jet cross-sections in QCD, Nucl. Phys. B 507 (1997) 295-314, http://dx.doi.org/10.1016/S0550-3213(97)00574-9, arXiv:hep$\mathrm{ph} / 9706545$.

[20] V. Hirschi, et al., Automation of one-loop QCD corrections, JHEP 05 (2011) 044, http://dx.doi.org/10.1007/JHEP05(2011)044, arXiv:1103.0621.

[21] G. Ossola, C.G. Papadopoulos, R. Pittau, Reducing full one-loop amplitudes to scalar integrals at the integrand level, Nucl. Phys. B 763 (2007) 147-169, http://dx.doi.org/10.1016/j.nuclphysb.2006.11.012, arXiv:hep-ph/0609007. 
[22] G. Ossola, C.G. Papadopoulos, R. Pittau, CutTools: A program implementing the OPP reduction method to compute one-loop amplitudes, JHEP 0803 (2008) 042 http://dx.doi.org/10.1088/1126-6708/2008/03/042, arXiv:0711.3596.

[23] M. Ciccolini, A. Denner, S. Dittmaier, Electroweak and QCD corrections to Higgs production via vector-boson fusion at the LHC, Phys. Rev. D 77 (2008) 013002, http://dx.doi.org/10.1103/PhysRevD.77.013002, arXiv:0710.4749.

[24] M. Ciccolini, A. Denner, S. Dittmaier, Strong and electroweak corrections to the production of Higgs +2 jets via weak interactions at the LHC, Phys. Rev. Lett. 99 (2007) 161803, http://dx.doi.org/10.1103/PhysRevLett.99.161803, arXiv:0707.0381.

[25] G. Corcella, I. Knowles, G. Marchesini, S. Moretti, K. Odagiri, et al., HERWIG 6: An event generator for hadron emission reactions with interfering gluons (including supersymmetric processes), JHEP 0101 (2001) 010, http://dx.doi.org/10.1088/1126-6708/2001/01/010, arXiv:hep-ph/0011363.

[26] M. Bahr, S. Gieseke, M. Gigg, D. Grellscheid, K. Hamilton, et al., Herwig++ physics and manual, Eur. Phys. J. C 58 (2008) 639-707, http://dx.doi.org/ 10.1140/epjc/s10052-008-0798-9, arXiv:0803.0883.

[27] T. Sjostrand, S. Mrenna, P.Z. Skands, PYTHIA 6.4 physics and manual, JHEP 0605 (2006) 026, http://dx.doi.org/10.1088/1126-6708/2006/05/026, arXiv:hep-ph/ 0603175.

[28] https://twiki.cern.ch/twiki/bin/view/LHCPhysics/CrossSections,

[29] A. Martin, W. Stirling, R. Thorne, G. Watt, Parton distributions for the
LHC, Eur. Phys. J. C 63 (2009) 189-285, http://dx.doi.org/10.1140/epjc/ s10052-009-1072-5, arXiv:0901.0002.

[30] M. Cacciari, G.P. Salam, G. Soyez, The anti- $k(t)$ jet clustering algorithm, JHEP 0804 (2008) 063, http://dx.doi.org/10.1088/1126-6708/2008/04/063, arXiv:0802.1189.

[31] M. Cacciari, G.P. Salam, G. Soyez, Fastjet user manual, Eur. Phys. J. C 72 (2012) 1896, http://dx.doi.org/10.1140/epjc/s10052-012-1896-2, arXiv:1111.6097.

[32] A. Martin, R. Roberts, W. Stirling, R. Thorne, Uncertainties of predictions from parton distributions. 1: Experimental errors, Eur. Phys. J. C 28 (2003) 455-473, http://dx.doi.org/10.1140/epjc/s2003-01196-2, arXiv:hep-ph/0211080.

[33] R. Frederix, S. Frixione, V. Hirschi, F. Maltoni, R. Pittau, et al., Four-lepton production at hadron colliders: aMC@NLO predictions with theoretical uncertainties, JHEP 1202 (2012) 099, http://dx.doi.org/10.1007/JHEP02(2012)099, arXiv: 1110.4738 .

[34] K. Hagiwara, Q. Li, K. Mawatari, Jet angular correlation in vector-boson fusion processes at hadron colliders, JHEP 0907 (2009) 101, http://dx.doi.org/ 10.1088/1126-6708/2009/07/101, arXiv:0905.4314.

[35] C. Englert, D. Goncalves-Netto, K. Mawatari, T. Plehn, Higgs quantum numbers in weak boson fusion, JHEP 1301 (2013) 148, http://dx.doi.org/ 10.1007/JHEP01(2013)148, arXiv:1212.0843.

[36] A. Djouadi, R. Godbole, B. Mellado, K. Mohan, Probing the spin-parity of the Higgs boson via jet kinematics in vector boson fusion, arXiv:1301.4965. 